\title{
ARTHROPLASTY OF THE KNEE
}

\author{
A Follow-up Study \\ J. S. Speed and Philip C. Trout, Memphis, Tennessee* \\ From the Campbell Clinic, Memphis
}

In this paper the results of a follow-up study of fascial arthroplasties of the knee joint which have been performed at the Campbell Clinic are presented, together with generalisations regarding the operation which from our experience seem justified. Ankylosis of the knee is not a crippling deformity and for this reason most orthopaedic surgeons agree that a knee which is stiff in good functional position is preferable to the uncertainties of arthroplasty. It is the authors' opinion, based upon this study, that in properly selected cases arthroplasty of the knee offers a practical method of relief from a disabling deformity which is a constant source of annoyance and embarrassment, particularly in women. In such cases, the patient should have a reasonable expectation of obtaining: 1) between 60 and 90 degrees of active movement with adequate muscular control; 2) sufficient stability to permit ordinary activity without the use of external support; 3) no pain, or pain of such minor degree as not to limit an average day's work in occupations other than those involving manual labour.

\section{INDICATIONS FOR ARTHROPLASTY OF THE KNEE}

Arthroplasty is indicated in patients with fibrous or bony ankylosis of the knee, between the ages of bone maturity and fifty years, who are not required to perform manual labour and in whom the ankylosis is the result of: 1) acute infective arthritis of haematogenous origin; 2) suppurative arthritis from punctured wounds or open fractures, the infection having been limited to the joint and unassociated with osteomyelitis; 3) trauma followed by incomplete painful ankylosis or complete ankylosis, wherein the bony components of the joint are not materially disturbed.

Formerly, most arthroplasties were performed for ankylosis from acute infective arthritis, usually of a gonorrheal nature, or punctured wounds of joints. Recent advances in the treatment of acute infections by sulphonamide drugs and antibiotics has considerably reduced the incidence of ankylosis from these sources.

\section{CONTRA-INDIGATIONS TO ARTHROPLASTY OF THE KNEE}

Arthroplasty is contra-indicated when ankylosis of the knee is incident to, or associated with: 1) multiple rheumatoid arthritis;2) tuberculosis; 3) osteomyelitis, either haematogenous or associated with open fractures; 4) obesity; 5) osteoporosis. Although we still advise arthroplasty of the knee for certain severely disabled patients with multiple ankyloses from rheumatoid arthritis, the purpose of the operation is not the same, nor do we expect as good results as in the favourable group. Osteoporosis provides a poor type of bone for the reconstruction of a weight-bearing joint. Erosion and absorption of the articular surfaces often cause instability, and valgus or varus deformities. The operation should be postponed, and active weight-bearing encouraged, until recalcification is adequate.

One hundred and seventy-six fascial arthroplasties of the knee were performed by various members of our staff before 1947. In the beginning, the indications and contraindications were not clearly understood, and the operation was performed upon many patients who, to-day, would not be considered suitable subjects. Such cases have been excluded from this study, not with the object of improving end-result statistics but simply because they

* Paper read at the combined meeting of the American, British, and Canadian Orthopaedic Associations in Quebec, June 1948, with cinematographic demonstration.

vol. $31 \mathrm{~B}$, No. 1, FEBRUARY 1949 
did not contribute to the solution of the fundamental problem-that is to say, the value of the operation in the type of case which we now regard as appropriate for fascial arthroplasty.

The early arthroplasties, wherein chromicised pig's bladder was used, were failures. Of four cases in which a single fixed vitallium mould was placed over the lower end of the femur, only one was successful. The patients in these two groups are not included in the present review. The failures after the use of the vitallium mould are explained by the fact, as shown by Smith-Petersen, that if a foreign body mould is to function satisfactorily it must be freely movable within the joint; fixation to either articular surface defeats the purpose of the mould. In the four cases in question, an active fibrous tissue and bony irritative reaction took place, the free surface of the mould becoming partly covered with a pannus-like formation of new bone. It was hoped that early removal of the mould might terminate this reaction, but in only one of the four cases was the process arrested before ankylosis recurred.

The development of a satisfactory mould should increase the number of good results in that group of cases in which we now consider that arthroplasty of the knee joint is indicated, and perhaps widen the scope of the operation to include a large group of patients with ankylosis of the knee due to rheumatoid arthritis.

\section{BIOLOGIGAL AND ADAPTIVE GHANGES AFTER ARTHROPLASTY}

Our experience with fascial arthroplasties of both hip and knee joints has led us to the conclusion that certain biological and adaptive changes are constantly taking place, so that one can never say that an end-result has been achieved. Nevertheless, follow-up studies have shown that, after fascial arthroplasty of the hip joint, function usually deteriorates gradually over a period of years, whereas, after arthroplasty of the knee joint, function reaches its maximum after five to ten years and subsequently remains remarkably stable. For this reason, a follow-up period of at least five years is believed to be necessary for proper estimation of the functional results.

In view of the fact that many real or apparent failures manifest themselves within the first five years, a conscientious effort has been made to trace by correspondence or re-examination every patient who had a fascial arthroplasty and who was considered suitable according to our present criteria. Of the patients in this group, sixty-five were traced for five or more years after operation; one hundred and eleven were not traced, or were considered unsuitable.

The pathological processes responsible for ankylosis in the sixty-five suitable cases were of three types: Neisserian infection; haematogenous infection other than Neisserian suppurative arthritis; and traumatic arthritis. Until recent years, gonorrheal arthritis was the most common cause of monarticular ankylosis, and the infection was never reactivated at the time of operation. If the etiological factor was a blood-borne infection other than gonorrhea, the pathological process was classified as infective arthritis. Ankylosis from suppurative arthritis includes those cases in which the infecting organism, usually the staphylococcus or streptococcus, was introduced directly into the joint by a penetrating wound or surgical arthrotomy. In this group, the articular cartilage was destroyed rapidly, thus giving rise to early ankylosis. Injury without infection, except in the elbow, is seldom responsible for complete ankylosis. Nevertheless, arthroplasty is often indicated for the relief of pain in an injured joint with incongruous surfaces, or one in which movement is blocked because of comminution of the articular surfaces.

\section{SURGICAL TREATMENT}

The Campbell technique of arthroplasty of the knee has been employed exclusively in this group of cases. Some of the fundamental principles which experience has proved to be sound are: 1) The simplest type of hinge joint should be constructed, with single broad 


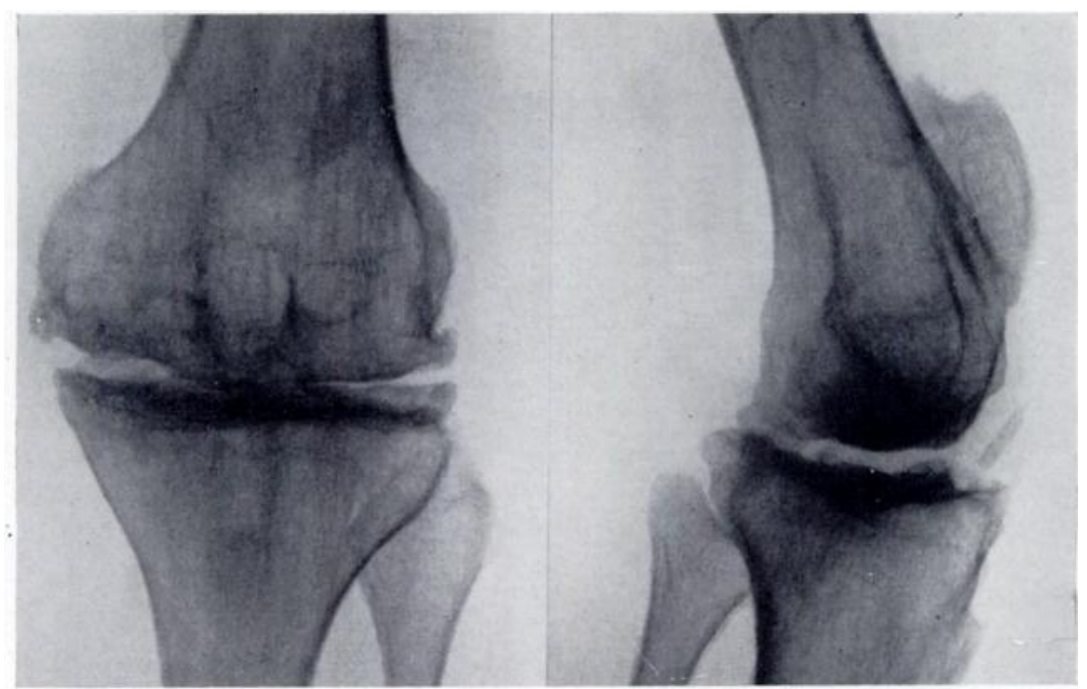

Case 1. Radiograph twenty-three years after arthroplasty of knee.

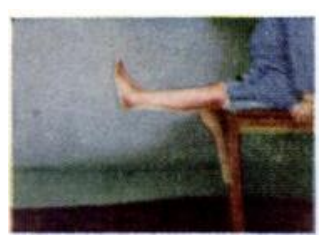

A

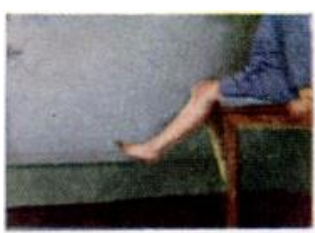

B

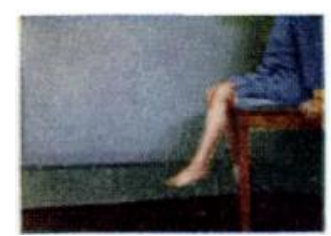

C

Case 1. Range of movement from 180 to 110 degrees.

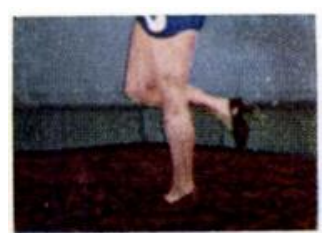

$\mathrm{D}$

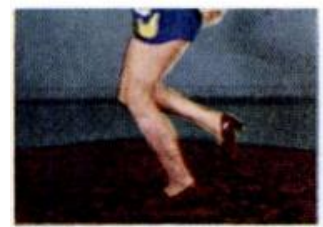

E

D, E - Weight-bearing in extension and flexion.

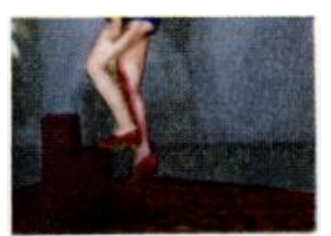

F

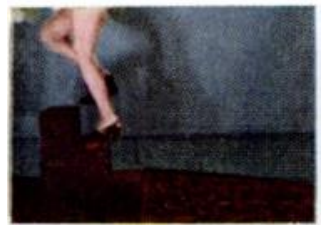

G

F, G-Ascending stairs in normal stride.

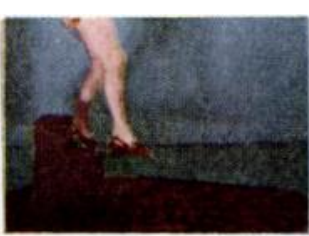

H

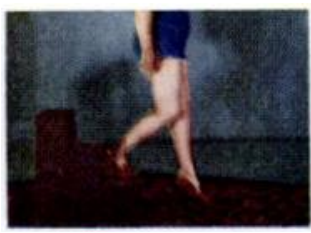

I

$\mathrm{H}, \mathrm{I}$-Descending stairs in normal stride.

Fig. 1

Case 1. Fascial arthroplasty of the left knee for bony ankylosis due to acute infective arthritis, re-examined twenty-three years after operation. The radiographs are shown above. There is 70 degrees of movement from 180 to 110 degrees $(\mathrm{A}, \mathrm{B}, \mathrm{C})$. The knee is stable and free from pain $(\mathrm{D}, \mathrm{E})$. The patient can go up and down steps normally (F, G; H, I) and she can walk eight or ten miles a day. 
femoral and tibial condyles. The formation of a tibial spine (Putti) or a wedge-shaped femoral condyle (Haas) increases the likelihood of incongruity of the articular surfaces, since they require that nature shall accomplish by adaptive processes a relationship between the articular surfaces which can be achieved at operation. 2) The extensor mechanism should be disturbed as little as possible; hence, division of the quadriceps tendon or detachment of the insertion of the patellar tendon is unwise. Adequate exposure may be obtained without either of these procedures. 3) Sufficient bone should be removed from the posterior parts of the femoral condyles to permit free movement of the tibia well up into the popliteal space. 4) In ankylosis of one-half the joint, the other half being relatively normal and with intact articular cartilage, a hemi-arthroplasty limited to the ankylosed side is contra-indicated. Without exception, this procedure has been a failure. 5) The use of a fascial covering of the new joint may not be necessary, but it is of value in keeping the bone surfaces separated until they are covered with fibrocartilage. 6) The ultimate stability of the joint depends upon: $a$ ) wide weight-bearing surfaces of the tibia and femur; $b$ ) ligamentous and muscular support. The collateral ligaments and joint capsule should be stripped carefully from the bone and preserved. The cruciate ligaments, if present, must be sacrificed.

\section{RESULTS OF ARTHROPLASTY}

The reconstruction problem and the operative technique are identical in fibrous and bony ankylosis. The practical value of an arthroplasty to the patient must be judged by the function obtained. One can form a true estimate of the functional capacity only by observing the patient walk, and go up and down steps, and by knowing how well the joint withstands the demands of an ordinary day's work. Printed words and tables cannot convey this information; even the moving pictures which accompanied presentation of this article were inadequate to demonstrate the satisfaction which 70 per cent. of these patients derived from the operation. The estimate of results was based upon the range of movement, stability, position of the knee in extension, and the amount of pain which the patient experienced with average use of the knee.

Range of movement-An arthroplasty of the knee is most efficient and durable if the range of movement gained is between 70 and 90 degrees (from 180 degrees to between 110 degrees and 90 degrees). Movement beyond 90 degrees may produce instability. Sixty degrees gives a good functional joint, except for going up and down stairs. Movement of 40 to 60 degrees is considered fair; patients with this range can walk without a limp and sit in a chair without being conspicuous. If movement is less than $\mathbf{4 0}$ degrees, the result is regarded as poor. Nevertheless, all patients in this group who failed to obtain the maximum range of movement preferred even the limited range to a stiff joint, and none wished to be deprived of the mobility which had been gained. Analysis of the sixty-five cases showed that the range of movement was 60 degrees or more in half the cases (Table I).

Stability-Stability is both an anatomical and a functional equation. Good stability is that degree which permits ordinary activity. Most patients in this study had a little increase in lateral or antero-posterior mobility when the joint was flexed; but all had good stability in extension with the quadriceps contracted. In those who were regarded as having fair stability there was some definite abnormal mobility and the joint was incompletely stabilised by quadriceps contraction. These patients found it necessary to be careful while walking, but required no external support. Patients who complained that their knees often "gave away," and those who had such abnormal mobility as to necessitate external support were regarded as having poor stability. On this basis it was found that two-thirds of the cases gained good stability; in one-third there was fair or poor stability or reankylosis (Table II).

Alignment when weight-bearing-The position of the extended knees in weight-bearing showed normal alignment in half the cases, some valgus deformity in nearly one-third, and varus or recurvatum in a few cases (Table III). 
Pain-It was interesting to observe how few patients had pain. There was often considerable grating on active movement of the joint; and such irregularity of the articular surfaces was demonstrated, not only on physical but on radiographic examination, that one might well have expected movement of the knee to be extremely painful. As shown in Table IV, however, pain was no real handicap.

TABLE I

RANGe OF MOVEMENT AFTER ARTHROPLASTY

\begin{tabular}{|c|c|c|c|}
\hline & & & Cases \\
\hline \multirow[t]{2}{*}{$\begin{array}{l}\text { Good }\left(60^{\circ} \text { or more }\right) \\
\text { Fair }\left(60^{\circ} \text { to } 40^{\circ}\right) \\
\text { Poor }\left(40^{\circ} \text { or less }\right) \\
\text { Reankylosis }\end{array}$} & \multirow[t]{2}{*}{$\cdot \dot{\cdot} \cdot$} & $\begin{array}{r}36 \\
12 \\
9 \\
8\end{array}$ & $\begin{array}{l}55 \cdot 4 \% \\
18 \cdot 5 \% \\
13.8 \% \\
12 \cdot 3 \%\end{array}$ \\
\hline & & 65 & $100.0 \%$ \\
\hline
\end{tabular}

TABLE III

Weight-bearing Alignment after ARTHROPLASTY

\begin{tabular}{|c|c|c|}
\hline & & Cases \\
\hline \multirow[t]{2}{*}{$\begin{array}{lllll}\text { Straight } & \cdot & \cdot & \cdot & \\
\text { Valgus } & \cdot & \cdot & \cdot & : \\
\text { Varus } & : & \cdot & \cdot & : \\
\text { Recurvatum } & & \cdot & & \\
\text { Not included } & \text { (reankylosis) }\end{array}$} & $\begin{array}{r}32 \\
19 \\
4 \\
2 \\
8\end{array}$ & $\begin{array}{r}49 \cdot 0 \% \\
30 \cdot 0 \% \\
6.0 \% \\
3.0 \% \\
12.0 \%\end{array}$ \\
\hline & 65 & $100 \cdot 0 \%$ \\
\hline
\end{tabular}

TABLE II

Stability of THE KNEE AFTER ARTHROPLASTY

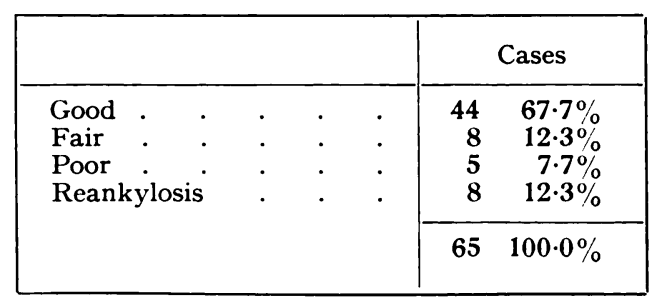

TABLE IV

Pain after Arthroplasty

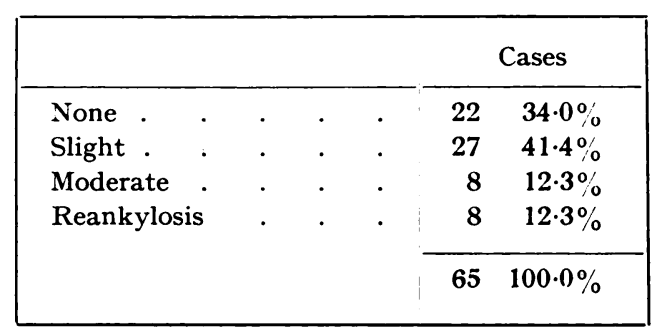

TABLE V

Results of Arthroplasty of the KNeE

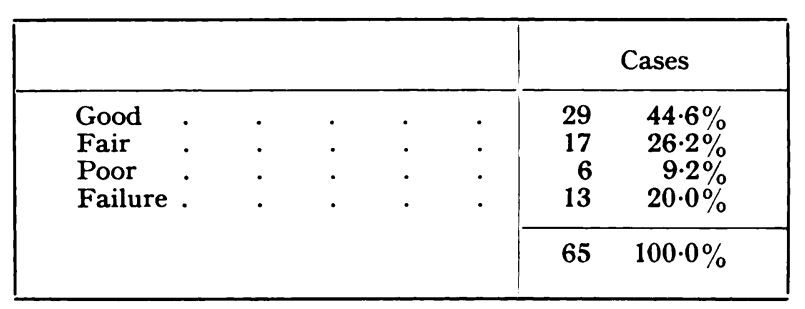

\section{COMPOSITE ESTIMATE OF RESULTS}

Patients who were regarded as having gained a good result had a range of movement of 60 degrees or more, slight or no pain, adequate stability, and a good weight-bearing position of the joint. Those with fair results had from 40 to 60 degrees of movement, with or without moderate pain, adequate stability and a good weight-bearing position. The results were considered to be poor in patients who had less than $\mathbf{4 0}$ degrees of movement, or in whom there was instability, or a faulty weight-bearing position of the joint. The operation was classified as a failure in those who had recurrence of ankylosis or for whom surgical reankylosis was necessary because of instability and pain (two cases).

It is appreciated that the personal equation of the examiner is an important element in final estimation of results. A sincere effort has been made to assess accurately the results of the fascial arthroplasties of the knee which we have performed in the sixty-five cases considered suitable for the operation. These results are shown in Table V.

VOL. $31 \mathrm{~B}$, No. 1, FEBRUARY 1949 


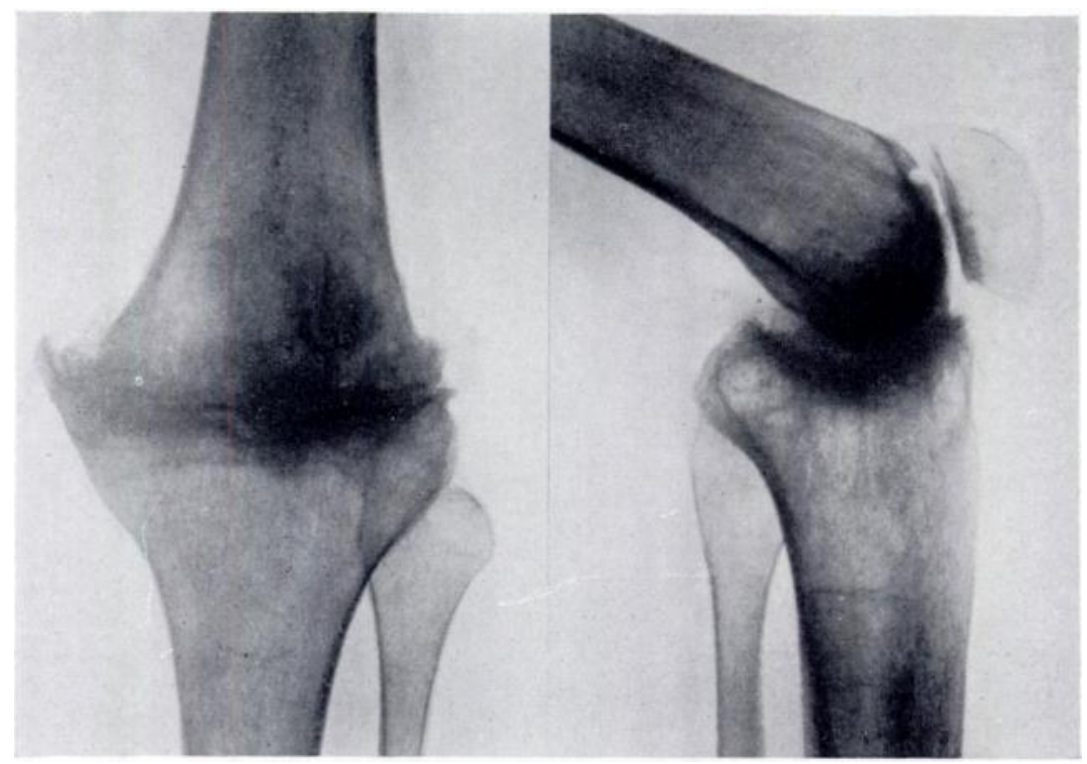

Case 2. Radiographs twenty-one years after arthroplasty of knee.

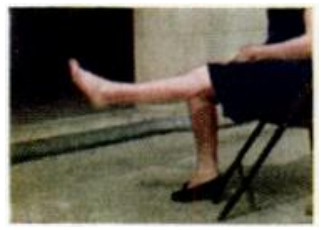

$A$

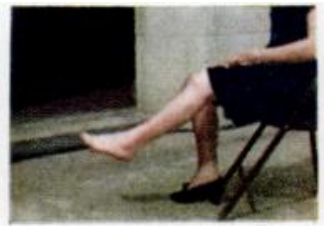

B

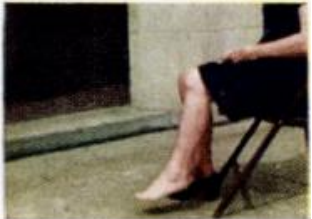

$\mathrm{C}$

Range of movement from 180 to 100 degrees.

FIG. 2

Case 2. Fascial arthroplasty of the left knee for bony ankylosis due to acute infective arthritis, followed-up twenty-one years after operation. The radiographs are shown above. There is active and passive movement from 180 to 100 degrees (A, B, C). She carries on with all ordinary physical activities including standing at work for eight hours a day and has no instability and no pain.

\section{SUMMARY}

1. Arthroplasty of the knee joint should be performed only in carefully selected cases. Criteria for the operation are outlined.

2. In our experience, 70 per cent. of properly selected patients secure good or fair results. An additional 12 per cent., whose anatomical or functional results were classified as poor, preferred the movement which had been gained to ankylosis of the joint.

3. The major functional adaptation of the knee joint takes place during the first five years after arthroplasty. Several patients who had a poor range of movement after one or two years developed an excellent range by the end of five years.

4. Instability, when present, usually became apparent within the first five years.

5. Joints which were still stable at the end of five years usually remained so over a long period of time. Four patients have been traced for twenty to twenty-five years, and three have been traced for over twenty-five years.

6. Since the incidence of ankylosis of the knee joint from gonococcal and pyogenic infections has been reduced by the use of antibiotics, fewer patients are suitable subjects for arthroplasty. 


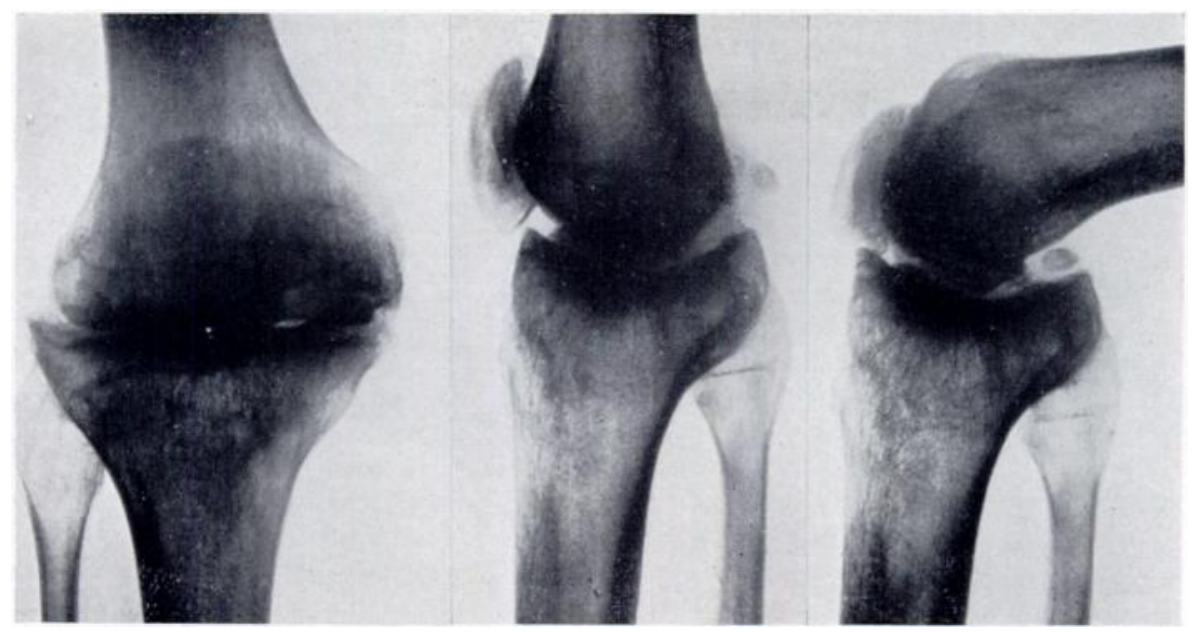

Case 3. Radiographs fifteen years after arthroplasty of knee.

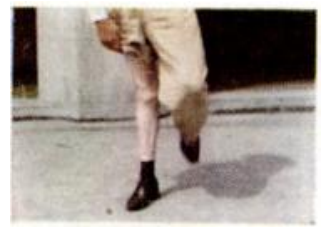

A

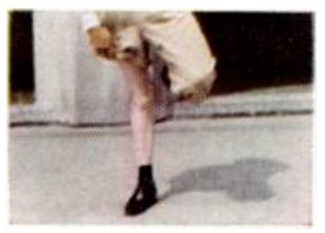

$\mathrm{B}$

Case 3. Weight-bearing on affected limb, showing stability.

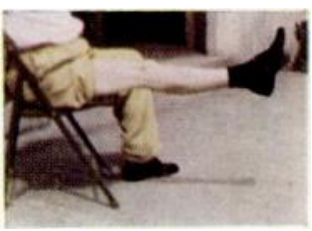

(

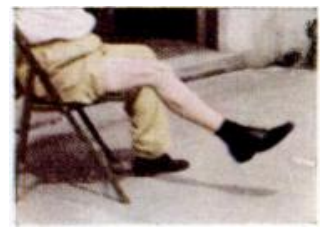

$\mathrm{D}$

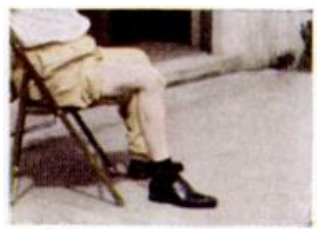

F

Range of movement from 180 to 90 degrees.

FIG. 3

Case 3. Fascial arthroplasty of right knee for fibrous ankylosis due to acute infective arthritis, followed-up fifteen years after operation. The radiographs are above. There is excellent stability $(\mathrm{A}, \mathrm{B})$ and movement from 180 to 90 degrees $(C, D, E)$. This patient did not secure the maximal range of movement until ten years after operation.

\section{DISCUSSION}

Sir Reginald Watson-Jones (London, England)-I feel sure that the reason I have been invited to discuss these papers from two famous clinics-the Campbell clinic, and the clinic of Dr Samson in Montreal-must be that I am known to be a staunch exponent of arthrodesis rather than arthroplasty of the weight-bearing joints. It is all very well for a patient who has been subjected to arthroplasty to sit in a chair and demonstrate movement-but can he walk ten miles; and can he jump from a five-barred gate? These are the criteria which I demand; and no doubt it was thought wise that discussion should be sought from an unbeliever. I would admit at once that whereas complete success in arthrodesis of the ankle joint depends on free mobility of the subastragaloid and mid-tarsal joints; and whereas success in arthrodesis of the hip joint demands free mobility of the pelvis, and of the opposite hip, so that the arthrodesed joint does not appear to be stiff at all; arthrodesis of the knee joint causes obvious stiffening which cannot be concealed. No neighbouring joint is available to take over function, and the disability is indeed very great.

VOL. 31 B, NO. 1, FEBRUARY 1949 
I have known patients to say that they would prefer amputation and an artificial limb to complete stiffness of the knee joint. If there ever was indication for arthroplasty of a weight-bearing joint it would be in the knee.

Unfortunately, however, the problem of arthroplasty of the knee joint is infinitely greater than that of arthroplasty of the hip. When the purpose of a mobilising operation is to gain movement in every direction, as in the case of the ball-and-socket joint of the hip, it can be secured with relative certainty by ruthless excision of bone, burning of raw surfaces, and interposition of a metal cap of rounded shape. But when the purpose of operation is to secure free movement in one direction, and perfect stability in the other, the problem is entirely different. It must be acknowledged that success in operative mobilisation of the hip joint-limited as it may be-has not yet been equalled, or even approached, by operative procedures which hope to achieve stable mobilisation of the knee joint.

The careful studies of Dr Boyd, Dr Speed, Dr Trout, and Dr Samson must lead us to one conclusion. Even in the hands of these great experts, and even in carefully selected cases-selected not only by reason of the type of ankylosis, and the ability of the patient to co-operate in difficult and painful after-treatment, but also by reason of the limited activities which the patient proposes to pursue-no more than about 50 per cent. of cases are successful. There can be no doubt that this procedure is still in an experimental stage. It is most important that the pioneer work of the Campbell clinic should continue; and we hope that Dr Samson's results will be recorded in even more accurate detail. But when it is recognised that after careful selection, and in the hands of experts, success is to be expected in no more than about half the cases, is it not wise for those of us who are less expert and less experienced to hesitate?

My own practice has been to reserve arthroplasty of the knee joint for patients with multiple ankyloses -ankylosis of both knee joints, and ankylosis of the knee and hip joints of the same limb. I am quite prepared to believe that some day the pioneers will prove a much wider application than this; but meanwhile, conservatism may be justified. We know the limitation of arthrodesis; but at the same time we are sure of its success; and it is not every patient who is prepared to accept the pain of operation, and the discomfort of after-treatment, without some definite promise of the end-result which is to be achieved. Are we, as yet, in a position to make such promise for arthroplasty of the knee joint?

Dr Joseph Freiberg (Cincinnati, Ohio)-I wish to show the records of a patient, fifteen years after arthroplasty of the knee for ankylosis due to acute infective arthritis after compound fractures of the upper third of the tibia, associated with extensive thrombo-phlebitis of the limb. In 1933, an arthroplasty of the Campbell type, which I have long preferred, was performed. This man is now forty-one years old and he is still playing tennis. There is $\mathbf{8 0}$ degrees of painless, stable movement. He is more than enth usiastic about his knee joint. The excellence of the result is shown in these slides. I have a number of other good arthroplasties of the knee, but this particular case is presented in order to show that when carefully done, and in well-selected cases, the operation is well worth while.

Dr Mather Cleveland (New York)-We are impressed with the "movies" which have been assembled by $\mathrm{Dr}$ Speed; they show beautiful end-results. Dr Samson's end-results, too, are impressive. But when you consider that Dr Speed is reporting no more than one-third of all knee joints in which arthroplasty has been attempted, discarding two-thirds for various reasons; and that less than 50 per cent. of those which are reported have yielded good, or better, results; I believe that we must pause for a word of caution. This operation may be a booby-trap for the unwary surgeon. Having served as consultant in the Armed Forces, and having all too often observed the enthusiasm with which a young man may be led into trouble by the written word, I believe that it would be wise for Dr Speed and Dr Samson to stress the difficulties, and perhaps talk more about the possibilities of failure than about the beautiful results which are all too infrequent. I hope that both these authors will indicate the pitfalls, and the dangers which are inherent in these surgical procedures, as well as the advantages which may be gained when there is satisfying success. Perhaps at another time they will show us moving pictures of one or two of their failures.

Dr Harold Boyd (Memphis, Tennessee, for Dr Speed)-I am sorry that the absence of Dr Speed in Hawaii has made it impossible for him to reply in person. Sir Reginald does not favour arthroplasty of the knee joint because patients are not able to run ten or twelve miles and jump from five-barred gates. But we must not ask too much of arthroplasty; many of us, who think that we are normal, cannot perform such feats; and it is not the purpose of this operation to improve the athletic abilities of the patient. It is true that arthrodesis of the knee joint is better suited to many cases than arthroplasty-and indeed we perform arthrodesis more often than arthroplasty-but this does not imply that in properly selected cases arthroplasty does not have its place. Stability is important, and we respect it in our British friends, but in this country we may perhaps be allowed to think that stability alone is not enough.

Significant points were discussed by Dr Bennett, Dr Cleveland, and Dr Freiberg. May I say that it was not the purpose of this contribution to dissuade surgeons from arthrodesis of the knee joint in favour of arthroplasty? The problem was presented to this Association in former years by Dr Campbell, and it was believed that the findings of late follow-up study should be recorded. We have no doubt that in properly selected cases the operation has a definite place in orthopaedic surgery, and that these suitable cases are entitled to the benefits which can be derived from the procedure. 